RESEARCH ARTICLE

\title{
Correlation Between Traffic Granularity and Defragmentation Periodicity in Elastic Optical Networks
}

\author{
Pouria Sayyad Khodashenas*, Jaume Comellas, Jordi Perelló, Salvatore Spadaro \\ Advanced Broadband Communications Center (CCABA), Universitat Politècnica de Catalunya (UPC), Jordi Girona 1-3, Barcelona, \\ 08034, Spain
}

\begin{abstract}
While elastic optical network technologies have emerged as promising solutions for future ultra-high speed optical transmission, the unavoidable spectral fragmentation problem that appears in such networks significantly degrades their performance. In light of this, spectral defragmentation technologies have been introduced in elastic optical networks, aiming to increase the spectrum utilization. During the defragmentation operation, the available fragmented spectrum bands are consolidated by re-allocating existing connections, either re-routing them along alternative routes and/or retuning them onto different spectrum portions. Obviously, spectral defragmentation increases network complexity and cost. Therefore, it is highly desirable to limit its application as much as possible, while keeping network performance within acceptable margins. In this paper, we focus on analyzing the correlation between the optimal (i.e., minimum) spectrum defragmentation periodicity in the network with the granularity of the supported traffic. For this purpose, we initially introduce a novel algorithm for efficient spectrum defragmentation. The proposed algorithm aims to consolidate the available fiber spectrum as much as possible, while minimizing the number of disrupted active connections. Then, supported on extensive simulation results, we show how spectral defragmentation periodicity can be effectively configured by having knowledge of the offered traffic granularity.

Copyright (c) 2013 John Wiley \& Sons, Ltd.

* Correspondence

Advanced Broadband Communications Center (CCABA), Universitat Politècnica de Catalunya (UPC), Jordi Girona 1-3, Barcelona, 08034, Spain.

E-mail: pkhodashenas@tsc.upc.edu
\end{abstract}

\section{INTRODUCTION}

Triggered by emerging services such as high-definition video distribution or social networking, the IP traffic volume has been exponentially increasing to date. Furthermore, the traffic growth rate will not stop here due to the day by day technology advances. For example, new hardware advances such as multi-core processing, virtualization and network storage will leverage innovative e-Science and grid applications requesting data flows of $10 \mathrm{~Gb} / \mathrm{s}$ up to terabit level. The predictable consequence is that network operators will require a new generation of optical transport networks in the near future, so as to to serve this huge and heterogeneous volume of traffic in a cost-effective and scalable manner [1]. In response to these large capacity and diverse traffic granularity needs of the future Internet, the elastic optical network architecture has been proposed [2]. By breaking the fixed-grid spectrum allocation limit of conventional wavelength division multiplexing (WDM) networ 2 astic optical networks increase the flexibility in the connection provisioning. To do so, depending on 
the traffic volume, an appropriately-sized optical spectrum portion is allocated to a connection in elastic optical networks. Moreover, unlike the rigid optical channels of conventional WDM networks, an elastic optical path can also expand or contract dynamically to meet different traffic loads [3]. As a result, incoming traffic demands can be served in a spectrum-efficient manne

This technological advance poses additional challenges on the networking level, and more specifically on the efficient connection establishment. Similar to WDM networks, an elastic optical connection must occupy the same spectrum portion between its end-nodes, that is, ensuring the so called spectrum continuity constraint. In addition, the entire bandwidth of the connections must be contiguously allocated, which is typically referred as the spectrum contiguity constraint. Upon tear down of connections, their allocated spectral resources are released, and could be assigned to new connection requests. In a dynamic traffic scenario, the random connection arrival and departure process in the network leads to the fragmentation of the spectral resources. Therefore, in medium and high loaded network scenarios, the possibility of finding sufficient contiguous spectrum to establish a connection over such fragmented spectrum can be very low, leading to connection request blocking despite having enough, but non-contiguous, spectrum resources. In light of this, there is an increasing demand from network operators to be able to periodically reconfigure their networks, aiming to improve the spectrum utilization [4]. This operation is called spectrum defragmentation.

During the defragmentation operation, the available fragmented spectrum bands are consolidated by reconfiguring active connections, i.e., changing their routes (nonhitless strategies), or assigning them a different portion of spectrum (hitless strategies) or both, while maintaining the imposed continuity and contiguity constraints [5]. However, the traffic granularity, namely, the average bit-rate per connection under a certain offered load to the network, has a direct effect on the spectrum fragmentation experienced at network links. Such effect can also be extended to the efficacy of a periodic spectrum defragmentation to keep an acceptable network performance. In particular, a deep analysis of the relationship between the traffic granularity and the defragmentation periodicity can allow a network operator to find the optimal defragmentation interval yielding the desired network performance, but

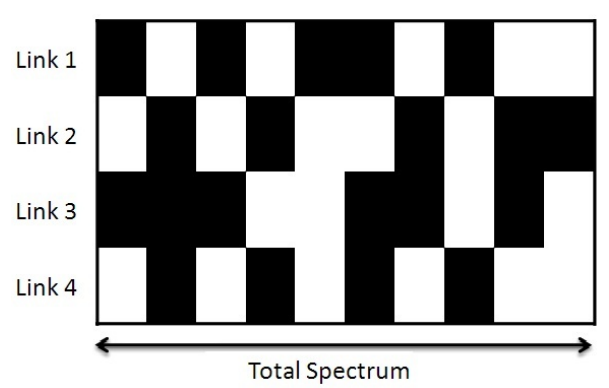

Figure 1. Scattered spectral fragments in four links of a typical elastic optical network.

requiring the minimum active connection disruptions and network control and management burden.

In this paper, we focus on this correlation between traffic granularity and defragmentation interval. To this end, we initially introduce a novel hitless defragmentation algorithm. The proposed algorithm maximizes the spectrum utilization in elastic optical networks. Then, we explore the impact of traffic granularity on the defragmentation interval through extensive simulation studies. The rest of paper is organized as follows. In Section II we initially review the related work on defragmentation operation. Section III details the proposed defragmentation algorithm. Simulation results are presented in Section IV. Finally, Section V concludes the paper.

\section{RELATED WORK}

As mentioned earlier, in a dynamic scenario, the spectral resources allocated to connections are released for future requests upon tear down. The randomness in the connection setup and tear down processes leads to fragmentation of the spectral resources in the network. Fig.1 shows scattered spectral fragments in four links of a typical elastic optical network. As shown, the available spectrum in the network links is fragmented into small non-contiguous spectral bands. The spectrum fragmentation significantly decreases the probability of finding enough contiguous spectrum for establishing new incoming connections, especially those traversing multi-hop paths (e.g., traversing links 1 to 4 in Fig.1) and/or requesting large amounts of bandwidth. In fact, new connection requests can be blocked in spite of 
having enough spectral resources if these are noncontiguous. Since an efficient utilization of the limited spectral resources in the network is an important issue for network operators, this problem has grabbed the research community attention. To address it, the proposal of spectral defragmentation strategies has appeared in the literature [5-13]. In general, spectral defragmentation means arranging the spectral resources in order to consolidate the available spectral fragments contiguously, so that they can be used for serving new incoming connection requests. Spectral defragmentation reduces connection blocking probability, and maximizes the service capacity of the network. In addition, it enables better network maintenance, more efficient network restoration and enhances the quality of service for a given spectral capacity.

For the first time, authors in [6] introduced the spectral defragmentation problem. They formulated it and proposed heuristic algorithms for spectral defragmentation operation. A scalable node architecture for realizing the spectral defragmentation proposal was also presented in [7] by the same authors. They showed a proof-ofprinciple experimental demonstration of spectral defragmentation in their studies. These studies clearly illustrated the benefits of spectral defragmentation in reducing the blocking probability of elastic optical networks. However, when the network has to be re-configured to perform defragmentation, existing active connections may be disrupted. Achieving a disruption-minimized spectrum optimization is a major challenge in spectral defragmentation. To address this, a disruption-minimized spectrum defragmentation algorithm in distance adaptive elastic optical networks was proposed [8], which uses a make-beforebreak re-routing scheme.

In addition, dynamic on-demand lightpath provisioning using spectral defragmentation was studied in [9]. A spectral defragmentation algorithm, as well as the corresponding node architectures with wavelength conversion to support on-demand lightpath provisioning was proposed. The same authors continued their work in [10] by introducing new heuristic defragmentation algorithms based on an auxiliary graph approach for elastic optical networks. Besides, a new routing and spectrum assignment algorithm which maximizes spectrum utilization in elastic optical networks was presented in [11]. Authors introduced a metric that quantifies the consecutiveness of the common available spectrum slots among relevant fibers. The first test-bed demonstration of an elastic optical network was presented in [12]. Authors used a real-time adaptive control plane for their test-bed that adjusts the modulation format and spectrum-positioning to maintain high QoS and spectral efficiency. Finally, a field-trial experiment of flexible spectrum switching and spectral defragmentation was presented in [13].

The aforementioned studies on the benefits of spectral defragmentation of elastic optical networks show a significant improvement in terms of spectrum efficiency and number of interrupted connections during the reconfiguration operation. However, there is no clear vision on the effects of traffic granularity on the defragmentation operation in a dynamic scenario. Authors in [14] focused on the problem from the defragmentation operation interval perspective. They proposed a defragmentation algorithm based on a factor that indicates the occupation of spectrum in a link or in the network. In this sense, by comparing the proposed factor with a given threshold, their algorithm is able to link the traffic granularity with the defragmentation interval. However, since the threshold is an arbitrary value in this study, and the whole available spectrum of network links is not considered for calculating the defragmentation factor, there is still a long way to go before really finding a correlation between traffic granularity and spectral defragmentation. In this work, we target at deeply analyzing this correlation. For this purpose, we initially propose a novel heuristic defragmentation algorithm, called iterative-defragmentation algorithm (IDA), which aims at increasing the network spectrum utilization, while minimizing the number of re-allocated connections during the defragmentation operation.

\section{IDA ALGORITHM}

The primary objective of the IDA algorithm is to maximize the consolidation of spectrum, followed by the secondary objective to minimize the number of re-allocated connections during the operation. To achieve the first objective, after establishing a certain number of connections over the network, we try to confine all existing connections in the lowest part of spectrum. Specifically, we process them one-by-one in descending 


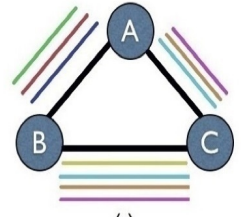

(a)

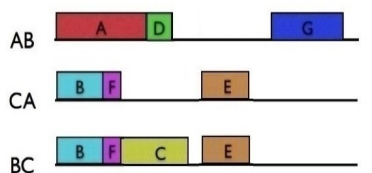

(c)

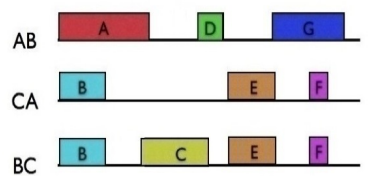

(b)

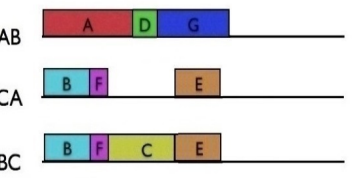

(d)
Figure 2. Illustrative Example: (a) 3-node simple network; (b) initial link spectral state; (c) link after the first iteration; (d) link spectral state after the second iteration.

order according to their position in the spectrum (i.e., connections allocated in the highest parts of the spectrum are processed first), also meeting the second objective in this way. The proposed algorithm is as follows:

\section{Iterative-Defragmentation Algorithm (IDA):}

1. Make a list of all the existing connections in the network and arrange them in descending order based on the connection operating spectrum.

2. Pick up the first connection from the ordered list and record its route in the network.

3. Check the spectrum status of the route in Step 2.

4. If a lower available portion of spectrum is found, re-allocate the connection (in a make-before-break fashion) in the lowest possible spectrum. Else, do not reconfigure the connection and go to Step 5.

5. Pick the next unprocessed connection in the list and repeat steps 2-4.

6. Repeat steps 1-5 for a number of iterations.

All possible existing connections in the network are re-allocated in the lowest available portion of spectrum on their route after the first iteration. However, since the generation of spectral fragments is a random process in a dynamic network scenario, which depends on many factors such as the offered load to the network, average bit-rate demand per connection or average path length, applying only one defragmentation iteration may not result in the higher spectrum consolidation. For example, consider the 3-node simple network shown in Figure 2(a) with the initial link spectral state shown in Figure 2-(b). As seen, there exists some connections in the network with different path length and bandwidth. The defragmentation operation tries to re-allocate existing connections in the lowest available portion of spectrum on their routes. Since some of these connections have smaller spectral requirements and short path length, there is more chance for them to be re-allocated (e.g., connections D or F). Meanwhile, as mentioned before, both continuity and contiguity spectrum constraints should be met when reallocating the connections, making some connections, such as E and G, to have lower defragmentation chances. In the event of defragmentation, existing connections are ordered as follows: G, F, E, D, C, A, B. According to the spectral status, connections $\mathrm{F}, \mathrm{E}, \mathrm{D}$ and $\mathrm{C}$ can be reconfigured on their paths since all links in their paths (for example (A, B) for connection D and (B, C) for connection C) have sufficient continuous spectrum available at a lower portion of the spectrum. However, connection $G$ cannot be re-allocated due to the spectral conflicts. As shown in 2-(c), the existing connections are confined after the first iteration; However, it is still possible to consolidate the connections even more. In the second iteration, the ordered list is updated as follows: G, E, C, D, A, F, B. Figure 2-(d) shows the spectral state of network, in which connections $\mathrm{G}$ and $\mathrm{E}$ are re-allocated to a lower spectrum portion. As shown, the second iteration optimally confines the existing connections in terms of spectral resources.

The number of re-allocations in the first iteration (Figure 2-(c)) is 4, while in the second iteration (Figure 2 -(d)) it is 2 . In general, by allowing more iterations in IDA, a more consolidated spectrum may be achieved, but at expenses of increasing the network control and management burden. Moreover, since the re-allocation of connections follows a make-before-break approach to avoid connection disruption, which is performed sequentially, the number of iterations impacts directly on the total defragmentation time. With this in mind, the effect of number of iterations on the performance of IDA will be investigated in the next section, together with the analysis of the correlation between traffic granularity and defragmentation periodicity in elastic optical networks.

\section{SIMULATION RESULTS}

To analyze the correlation between traffic granularity and defragmentation in elastic optical networks, we 


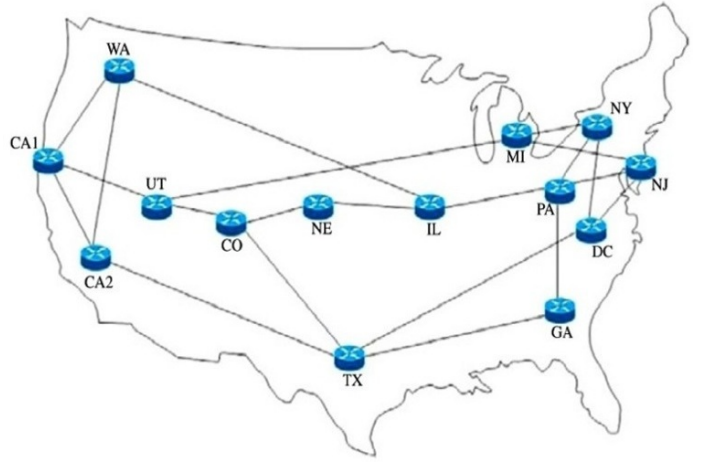

Figure 3. NSFnet topology.

simulate a network scenario where IDA defragmentation is performed on a periodic basis (hereafter referred as periodic defragmentation). Moreover, two additional scenarios are simulated for benchmarking purposes: 1) No-defragmentation, namely, a basic elastic optical network scenario without defragmentation capabilities; 2) On-demand defragmentation, where the defragmentation algorithm proposed in [9] is reactively executed upon blocking of an incoming connection request. Essentially, this algorithm tries to defragment the shortest path between the endpoints of the blocked incoming connection, reallocating the active connections supported on any link composing the shortest path (i.e., consolidating them to the lowest spectrum part, similarly as in IDA). Note that only the shortest path is defragmented here (e.g., in contrast to the network-wide periodic defragmentation) to avoid new incoming connections incur large establishment times. Regarding the RSA algorithm for allocating new incoming connections, all 3 scenarios run a typical k-Shortest Path with first-fit slot assignment [15].

The 14-node NSFnet topology (Figure 3) has been selected for the simulations. We assume a total optical spectrum of $1 \mathrm{THz}$ per link. This spectrum is discretized in units of $12.5 \mathrm{GHz}$, referred as frequency slots [2]. In addition, according to the asymmetric nature of today's Internet traffic unidirectional connections between sourcedestination nodes are considered. The offered load to the network follows a Poisson process, so that different offered loads are obtained by keeping the mean Holding Time (HT) of the connections constant to $200 \mathrm{~s}$, while modifying their mean Inter-Arrival Time (IAT) accordingly (i.e., offered load $=$ HT/IAT). Bit rate demands for each sourcedestination pair are randomly generated following an

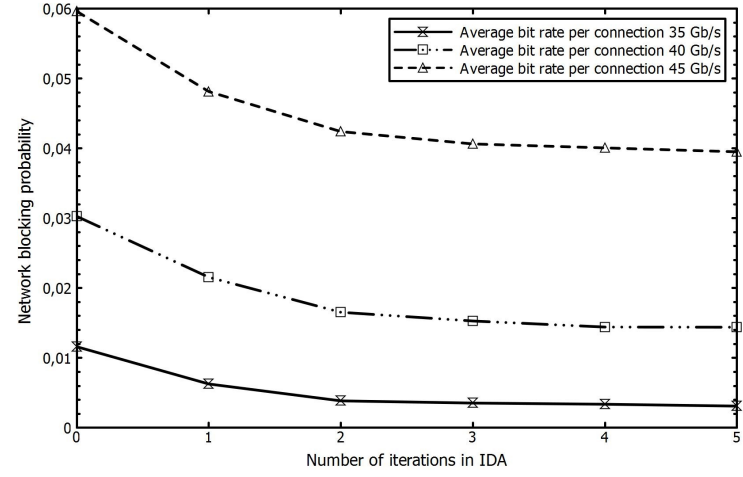

(a)

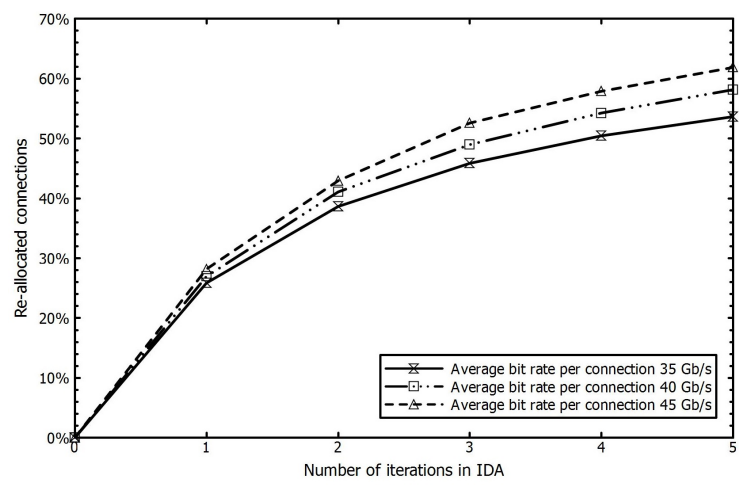

(b)

Figure 4. Effect of the number of iterations on the performance of the IDA algorithm.

exponential distribution over the range from $12.5 \mathrm{~Gb} / \mathrm{s}(1$ frequency slot) to $125 \mathrm{~Gb} / \mathrm{s}$ (10 frequency slot). Hence, we are implicitly considering a signal modulation format with spectral efficiency of $1 \mathrm{~b} / \mathrm{s} / \mathrm{Hz}$, like Binary Phase Shift Keying (BPSK) [16]. To start, we fix the defragmentation interval to 70 in the periodic defragmentation scenario. This means that a network-wide defragmentation is periodically performed every 70 connections established. Nonetheless, we will also evaluate the effect of this parameter on the network blocking probability later on in this section.

As a first study, we focus on periodic defragmentation and initially investigate the effect of the number of iterations performed by the IDA algorithm. The aim of this study is to find such a number of iterations in IDA that effectively reduces the blocking probability in the network, while keeping reasonable the number of reallocated connections. As mentioned before, more reallocated connections imply additional network control and management burden, which may not be translated into actual blocking probability reduction. 
Specifically, we offer a load of 14 per node (i.e., 14 offered connections per node in average), which makes up a total offered load to the network of 196. Then, we extract the results for three different average bit-rates per connection, namely, $35 \mathrm{~Gb} / \mathrm{s}, 40 \mathrm{~Gb} / \mathrm{s}$ and $45 \mathrm{~Gb} / \mathrm{s}$, leading to an average traffic offered per node equal to 490 $\mathrm{Gb} / \mathrm{s}, 560 \mathrm{~Gb} / \mathrm{s}$ and $630 \mathrm{~Gb} / \mathrm{s}$ respectively. Figure 4-(a) shows the blocking probability in the network against the number of iterations in IDA. As seen, by increasing the number of iterations, defragmentations are more effective, which helps in decreasing the blocking probability in the network. This is particularly true for 1 and 2 iterations under all average connection bit-rates. From then on, however, blocking probability reduction becomes marginal with the number of iterations. In Figure 4-(b), we quantify the percentage of re-allocated connections over the total number of active connections in the entire network. As shown there, for instance, by changing the number of iterations from 2 to $3,10 \%$ more connections have to be re-allocated, while almost no improvement in terms of blocking probability is experienced. This trend also holds for larger number of iterations. Hence, from now on, we fix the number of iterations in IDA to 2, which allows to significantly reduce the blocking probability in the network with reasonable network control and management overhead.

Figure 5 compares periodic defragmentation against no defragmentation and on-demand defragmentation in terms of network blocking probability as a function of the offered load per node. In this study, the offered load per node ranges from 11 to 14.5 , with an average bit-rate per connection equal to $40 \mathrm{~Gb} / \mathrm{s}$. This is translated into an offered traffic per node ranging from from $440 \mathrm{~Gb} / \mathrm{s}$ to $580 \mathrm{~Gb} / \mathrm{s}$. From the results, we observe that periodic defragmentation outperforms no defragmentation and even on-demand defragmentation along the entire offered load range. While periodic defragmentation improvement against no defragmentation was quite expected, it was not against the on-demand defragmentation. In fact, the observed improvements can be explained by the following two reasons. First, the network-wide defragmentation performed in periodic defragmentation against the shortest path-constrained defragmentation in the on-demand case (i.e., mandated by the stringent setup delays of initially blocked incoming connections, which plead for fast defragmentation operations). Second, the defragmentation

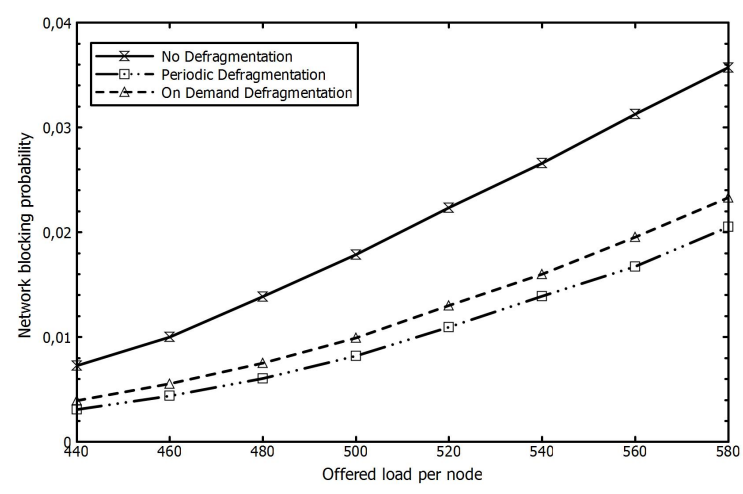

Figure 5. Network blocking probability for different traffic granularities. The offered load per node varies from 11 to 14.5 , with an average bit-rate per connection equal to $40 \mathrm{~Gb} / \mathrm{s}$.

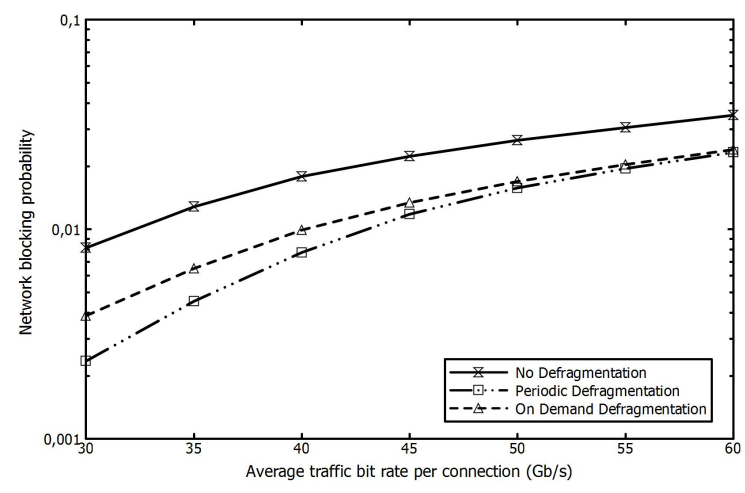

Figure 6. Network blocking probability for an offered traffic per node equal to $500 \mathrm{~Gb} / \mathrm{s}$. The average bit-rate per connection varies from $30 \mathrm{~Gb} / \mathrm{s}$ to $60 \mathrm{~Gb} / \mathrm{s}$, changing the offered load per node accordingly.

interval of 70 in periodic defragmentation. As will be shown later on, this parameter has crucial importance when comparing both defragmentation strategies.

To give further insight into the effects of the offered traffic characteristics on the benefits of network defragmentation, Figure 6 compares the network blocking probability achieved by periodic, on-demand and no defragmentation as a function of the average bit rate per connection. To produce these results, we have fixed an offered traffic per node of $500 \mathrm{~Gb} / \mathrm{s}$ in all cases. Hence, higher bit-rates per connection mean lower offered loads per node values and vice-versa. As illustrated in the figure, even under the same offered traffic, relative benefits of defragmentation are clearly affected by the actual bit-rates of the connections. For instance, for low bit-rates per connection, almost one order of magnitude blocking probability reduction is achieved by periodic 


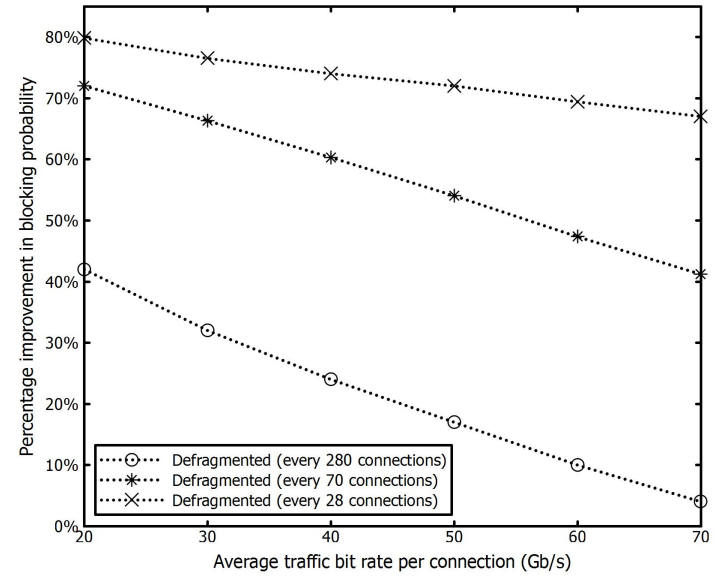

Figure 7. Percentage improvement in blocking probability for different bit-rates per connection (blocking probability equal to $2 \%$ with no defragmentation).

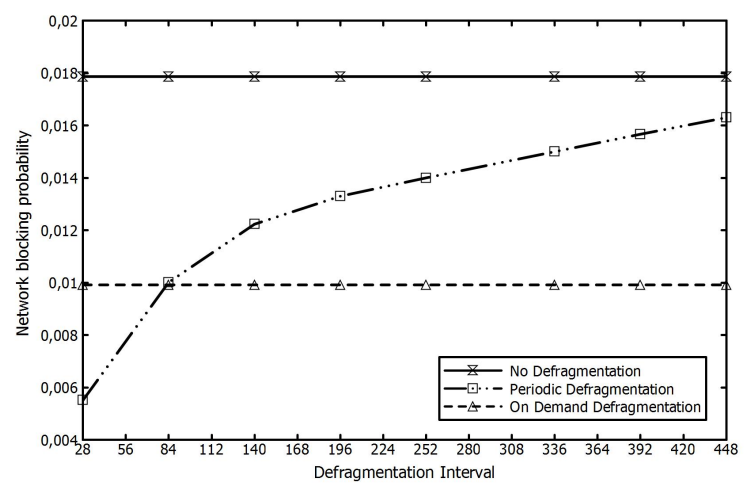

Figure 8. Network blocking probability against defragmentation interval in periodic defragmentation.

defragmentation against no defragmentation, while such a difference decreases when bit-rates increase. Comparing periodic versus on-demand defragmentation, we can also see that the former slightly outperforms the latter for low connection bit-rates. Again, this is due to the fact that network-wide defragmentation on a periodic basis (every 70 established connections) is more productive than reactive on-demand defragmentation constrained to the shortest path for time complexity reasons, especially with low bit-rate connections. Indeed, such differences decrease with the bit-rate per connection, as spectrally wider connections are more difficult to be efficiently reallocated in general.

As highlighted in previous figure, defragmentation benefits clearly depend on the bit-rate of those connections offered to the elastic optical network. To quantify these benefits, Figure 7 illustrates the profitability of defragmentation against no defragmentation for different bit-rates per connection. Only periodic defragmentation is considered here, with 3 different defragmentation intervals, namely, 28, 70 and 280. Furthermore, such a defragmentation profitability is measured as follows: for the evaluated bit-rate per connection we adjust the offered load per node in the network so as to obtain a $2 \%$ blocking probability $\left(\mathrm{BP}_{N D}\right)$ with no defragmentation. Then, we measure the improvement in blocking probability, equal to the steady-state blocking probability with no defragmentation minus the achieved blocking probability with periodic defragmentation $\left(\mathrm{BP}_{P D}\right)$, all divided by $\mathrm{BP}_{N D}$ :

$$
\text { Improvement }(\%)=\left[\left(B P_{N D}-B P_{P D}\right) / B P_{N D}\right] * 100
$$

Looking at Figure 7, the obtained results are very in-line with those in Figure 6, showing a periodic defragmentation improvement decreasing as the bit-rate of the connections increase. Moreover, the same behavior is observed no matter the applied defragmentation interval is, although smaller defragmentation intervals (i.e., more frequent defragmentation) seem to moderate this effect. For example, with a defragmentation interval of 280 , improvement sharply falls from around $40 \%$ in a network scenario with average connection bit-rate of $20 \mathrm{~Gb} / \mathrm{s}$, to merely $5 \%$ when connection bit-rate increases to $70 \mathrm{~Gb} / \mathrm{s}$, even though the offered load in both scenarios is adjusted to equally achieve $2 \%$ blocking probability. In contrast, with a defragmentation interval of 28 , ten times lower, improvement only decreases from $80 \%$ to around $70 \%$ when connection bit-rates change from $20 \mathrm{~Gb} / \mathrm{s}$ to $70 \mathrm{~Gb} / \mathrm{s}$, respectively.

Another conclusion that can be extracted from Figure 7 is the paramount importance of the defragmentation interval value on the improvements that periodic defragmentation can yield. Indeed, more frequent defragmentation foster improvement in blocking probability, although introducing more control and management burden in the network, as already mentioned in the paper. An interesting outcome of this figure, however, is that taking the bit-rate of the connections into account, a network operator can intelligently set the periodicity of the defragmentation in the network. For example, to achieve $40 \%$ improvement with a bit-rate of $70 \mathrm{~Gb} / \mathrm{s}$, a defragmentation interval of 70 is required. Nonetheless, if the bit-rate of the connections 
appears to be $20 \mathrm{~Gb} / \mathrm{s}$, the defragmentation interval can be increased to 280 , as only $1 / 4$ of the defragmentations are necessary in this scenario to achieve the same improvement. This could also open the possibility for operators to implement monitoring strategies of the incoming connections bit-rate (e.g., during pre-defined time periods or a number of connection establishments) to dynamically adjust the defragmentation interval in the network, making a good trade-off between blocking probability and control and management burden.

To round off the paper,Figure 8 shows in more detail the effect of the defragmentation interval on the performance of periodic defragmentation. To this end, we have offered a load of 12.5 per node with average bit-rate per connection of $40 \mathrm{~Gb} / \mathrm{s}$, which leads to blocking probability of $1 \%$ with on-demand defragmentation. In such a scenario, we plot the blocking probability versus defragmentation interval for no defragmentation, on-demand and periodic defragmentation. Of course, the former two behave constant with the defragmentation interval, as their performance does not depend on this parameter. However, we use them in the figure as a benchmark. As shown, when increasing the defragmentation interval, the possibility of successfully re-allocating connections in periodic defragmentation decreases. This could be explained from the fact that the randomness of the connection arrivals and departures creates such a bad network situation during each defragmentation period, that the defragmentation periodically performed cannot re-arrange it in a better way. In any case, periodic defragmentation always stays below no defragmentation for any of the evaluated intervals. Specifically, to get similar performance as on-demand defragmentation (or better), the defragmentation interval has to be set to 84 (or lower). This is the reason why periodic defragmentation achieved better performance than the on-demand one in Figure 5, where the defragmentation interval was set to 70. Therefore, provided that defragmentation intervals below 84 are acceptable for a network operator, periodic defragmentation can be a good option against the ondemand one, which has to be forcefully simpler to avoid incoming demands experiencing high establishment delays.

\section{CONCLUSION}

The recently proposed elastic optical network which provides enhanced flexibility in spectrum allocation and data rate accommodation has opened up a new prospect to serve the future Internet demands more efficiently. However, the inevitable spectral fragmentation problem in such networks significantly degrades their performance. To address the problem, the idea of spectral defragmentation in elastic optical network has been proposed. Assuming a periodic defragmentation scenario, this paper discussed the effects of the offered traffic characteristics (offered load and bit-rate per connection), as well as the defragmentation periodicity, on the profitability that defragmentation can yield to network operators. Indeed, while decreasing the defragmentation interval has direct impact on improving the blocking probability in the network, taking into account the bit-rate of the connections can also lead to the same results but with more relaxed defragmentation intervals and, thus, lower network control and management burden. All these findings were supported by extensive simulation results, where no defragmentation and ondemand defragmentation scenarios were also contemplated as a benchmark. For future work, authors will derive analytical models to formally quantify the effect of traffic granularity on the periodicity of defragmentation operation.

\section{ACKNOWLEDGEMENT}

This work has been supported by the Government of Catalonia and the European Social Fund through a FIAGAUR research scholarship grant and by the Spanish National project ELASTIC (TEC2011-27310).

\section{REFERENCES}

1. Cisco White Paper, "Cisco Visual Networking Index: Forecast and Methodology, 2011-2016".

2. M. Jinno, et al., "Spectrum-Efficient and Scalable Elastic Optical Path Network: Architecture, Benefits, and Enabling Technologies," IEEE Communications Magazine, vol. 47, no. 11, pp. 66-73, 2009.

Trans. Emerging Tel. Tech. 2013; 00:1-10 (C) 2013 John Wiley \& Sons, Ltd. DOI: $10.1002 /$ ett 
3. M. Jinno, et al., "Dynamic Optical Mesh Networks: Drivers, Challenges and Solutions for the Future," in Proc. ECOC, 2009.

4. K. Christodoulopoulos, et al., "Spectrally/Bitrate Flexible Optical Network Planning," in Proc. ECOC, Paper We.8.D.3, Sep. 2010.

5. R. Wang, et al., "Spectrum Management in Heterogeneous Bandwidth Networks," in Proc. Globecom, 2012.

6. N. Patel, et al., "Defragmentation of Transparent Flexible Optical WDM (FWDM) Networks," in Proc. OFC/NFOEC, 2011.

7. D. J. Geisler, et al., "Demonstration of Spectral Defragmentation in Flexible Bandwidth Optical Networking by FWM," IEEE Photonics Technology Letters, vol. 23, no. 24, pp. 1893-1895, 2011.

8. T. Takagi, et al., "Disruption Minimized Spectrum Defragmentation in Elastic Optical Path Networks that Adopt Distance Adaptive Modulation," in Proc. ECOC, 2011.

9. K. Wen, et al., "Dynamic On-demand Lightpath Provisioning Using Spectral Defragmentation in Flexible Bandwidth Networks," in Proc. ECOC, 2011.

10. Y. Yin, et al., "Dynamic on-demand defragmentation in flexible bandwidth elastic optical networks.," Optics express, vol. 20, no. 2, pp. 1798-804, Jan. 2012.

11. Y. Sone, et al., "Routing and Spectrum Assignment Algorithm Maximizes Spectrum Utilization in Optical Networks," in Proc. ECOC, 2011.

12. L. Paraschis, et al., "The First Testbed Demonstration of a Flexible Bandwidth Network with a Real-Time Adaptive Control Plane," in Proc. ECOC, 2011.

13. N. Amaya, et al., "Gridless optical networking field trial: flexible spectrum switching, defragmentation and transport of 10G/40G/100G/555G over 620-km field fiber," in Proc. ECOC, 2011.

14. X. Yu, et al., "Spectrum Compactness based Defragmentation in Flexible Bandwidth Optical Networks," in Proc. NFOEC, 2012.

15. K. Christodoulopoulos, et al., "Routing and spectrum allocation in OFDM-based optical networks with elastic bandwidth allocation", in Proc. Globecom, 2010.

16. M. Xia, et al., "Split spectrum: a multi-channel approach to elastic optical networking", Optics Express, Vol. 20, Issue 28, pp. 29143-29148, 2012.

\section{AUTHORS' BIOGRAPHIES}

Pouria Sayyad Khodashenas received his B.Sc. degree in 2005 from the University of Guilan, Rasht, Iran, and his M.Sc. degree in 2008 from University of Tabriz, Tabriz, Iran, both in electrical engineering. Since October 2009, he has been working towards a Ph.D. in telecommunications engineering at Universitat Politècnica de Catalunya (UPC, BarcelonaTech), Barcelona, Spain, being advised by Prof. Jaume Comellas. His research interests include all-optical communication systems, all-optical switches, nonlinear optics, quantum optics, multi-layer structures and semiconductor lasers, especially VCSEL.

Jaume Comellas received the M.S. (1993) and Ph.D. (1999) degrees in Telecommunications Engineering from Universitat Politcnica de Catalunya (UPC), Spain. His current research interests focus on IP over WDM networking topics. He has participated in many research projects funded by the Spanish government and the European Commission. He has co-authored more than 80 research articles in international journals and conferences. $\mathrm{He}$ is an associate professor at the Signal Theory and Communications Department of UPC.

Jordi Perellò received his M.Sc. and Ph.D. degrees in telecommunications engineering in 2005 and 2009, respectively, from UPC. Currently, he is an assistant professor in the Computer Architecture Department (DAC) at UPC. He has participated in various IST FP-6 and FP-7 European research projects, such as FP-7 projects STRONGEST, EULER, DICONET or NoE BONE, as well as FP-6 projects NOBEL Phase 2, e-Photon/One+ or COST Action 291. He has published more than 50 articles in international journals and conferences. His research interests concern resource management, qualityof-service issues, and survivability of future optical transport networks.

Salvatore Spadaro received the M.Sc. (2000) and the Ph.D. (2005) degrees in Telecommunications Engineering from Universitat Politècnica de Catalunya (UPC). He also received the Dr. Ing. degree in Electrical Engineering from Politecnico di Torino (2000). He is currently Associate Professor in the Optical Communications group of the Signal Theory and Communications Dept. of UPC. Since 2000 he is a staff member of the Advanced Broadband 
Communications Center (CCABA) of UPC. His research interests are in the fields of all-optical networks with emphasis on network control and management, resilience and network virtualization. 